\title{
Correlated electron systems on the Apollonian network
}

\author{
Andre M. C. Souza ${ }^{1,2}$ and Hans Herrmann 3,4 \\ ${ }^{1}$ Institut für Computerphysik, Universität Stuttgart, Pfaffenwaldring 27, 70569 Stuttgart, Germany \\ ${ }^{2}$ Departamento de Fisica, Universidade Federal de Sergipe, 49100-000 Sao Cristovao-SE, Brazil \\ ${ }^{3}$ Computational Physics, IfB, ETH Hönggerberg, HIF E 12, CH-8093 Zürich, Switzerland \\ ${ }^{4}$ Departamento de Física, Universidade Federal do Ceará, 60451-970 Fortaleza-CE, Brazil
}

(Received 4 October 2006; revised manuscript received 11 December 2006; published 20 February 2007)

\begin{abstract}
Strongly correlated electrons on an Apollonian network are studied using the Hubbard model. Ground-state and thermodynamic properties, including specific heat, magnetic susceptibility, spin-spin correlation function, double occupancy, and one-electron transfer, are evaluated applying direct diagonalization and quantum Monte Carlo techniques. In the strong-coupling limit, the quantum anisotropic spin-1/2 Heisenberg model is used and the phase diagram is discussed using the renormalization group method. The results support an antiferromagnetic order with a metal-insulator transition for $U / t=11.1$ at temperature $T=0$ and a tendency toward a nonordered phase in the half-filled Hubbard model for any $U / t$. We also indicate that the spectral dimension must control the magnetic behavior on the Apollonian network.
\end{abstract}

DOI: 10.1103/PhysRevB.75.054412 PACS number(s): 05.45.Df, 02.70.Ss, 71.10.Fd, 71.27.+a

\section{INTRODUCTION}

Scale-invariant networks have been the subject of intensive study in view of possible insights into inhomogeneous problems such as random magnets, surfaces, porous rocks, aerogels, sponges, etc. ${ }^{1,2}$ These structures can embody particular fractal substructures, like a scale-free network. In this case, the fraction of sites with $k$ connections follows a power law, ${ }^{3}$ which is very different from the usual topology of the Bravais lattice. Recently, the area of scale-invariant networks has been highly motivated by the creation of a synthetic nanometer-scale Sierpinski hexagonal gasket, a self-similar fractal macromolecule. ${ }^{4}$ New types of photoelectric cells, molecular batteries, and energy storage may be possible. This perspective raises questions about correlated electron systems on these networks, such as studies of quantum magnetism, superconductivity, metal-insulator transition, etc.

An important aspect that has to be analyzed is the influence of this topology on the properties of the Anderson metal-insulator transition (MIT) ${ }^{5,6}$ The Anderson MIT investigations on scale-free networks have shown that the spectral dimension characterizes the behavior of the MIT critical exponent, and the fractal dimension quantifies the critical disorder. Thus, the critical exponent on scale-free networks follows the mean-field prediction, and the localization of the electronic states is influenced by the connectivity of the network. ${ }^{5,6}$

These investigations suggest that the influence of these topologies may lead to a very novel and interesting behavior associated with the appearance of magnetic order on these networks. In particular, it would be interesting to investigate whether the decisive role for the magnetic behavior is the spectral or the fractal dimension.

In this paper, we investigate one family of free scaleinvariant graphs, the Apollonian network. ${ }^{7-9}$ The purpose is to examine correlated electrons on Apollonian networks. We explore the connection between the scale-invariant network topology and the magnetic properties of quantum magnetism models, namely, Hubbard and anisotropic spin-1/2 Heisen- berg models. We have examined the Hubbard model using the small-cluster diagonalization ${ }^{12,13}$ and quantum Monte Carlo techniques. ${ }^{14,15}$ The Heisenberg model is studied within a real-space renormalization group framework..$^{16-18}$

The construction of the Apollonian network has the topology of the contacts of an Apollonian packing of twodimensional disks. As initial configuration we use, for an Apollonian packing, three mutually touching circles inscribed inside a circular space. The interstices of the initial disks define a curvilinear triangle to be filled. In the next iteration, four disks are inscribed, each touching all the sides of the corresponding curvilinear triangle. This process is repeated indefinitely by setting disks in the newly generated curvilinear triangles. The Apollonian packing is a fractal whose dimension has been estimated as $1.3057 .{ }^{9}$

The Apollonian network (AN) is derived from the twodimensional Apollonian packing associating vertices with the circles and connecting two vertices if the corresponding circles touch. Figure 1 shows the first three generations of the AN. With this construction procedure one obtains a deterministic scale-free network where the number of sites at iteration $n$ is $\left(3^{n-1}+5\right) / 2$. Besides being a scale-free network, the AN has interesting properties, such as being Euclidean, matching, and space filling. ${ }^{7}$

The organization of this paper is as follows. The models are briefly introduced in Sec. II. The results for ground-state and thermodynamic properties of the Hubbard model on an AN are presented in Secs. III and IV, respectively. The phase diagram of the quantum anisotropic Heisenberg model is discussed in Sec. V and conclusions are presented in Sec. VI.

\section{MODELS}

Our study of the magnetic properties on an AN are based on the Hubbard model. The Hamiltonian of the Hubbard model is defined by

$$
\mathcal{H}_{u}=-t \sum_{\langle i j\rangle \alpha} c_{i \alpha}^{\dagger} c_{j \alpha}+U \sum_{i} n_{i \uparrow} n_{i \downarrow}
$$

where $c_{i \alpha}^{\dagger}, c_{i \alpha}$, and $n_{i \alpha} \equiv c_{i \alpha}^{\dagger} c_{i \alpha}$ are, respectively, the creation, anihilation, and number operators for an electron with spin $\alpha$ 


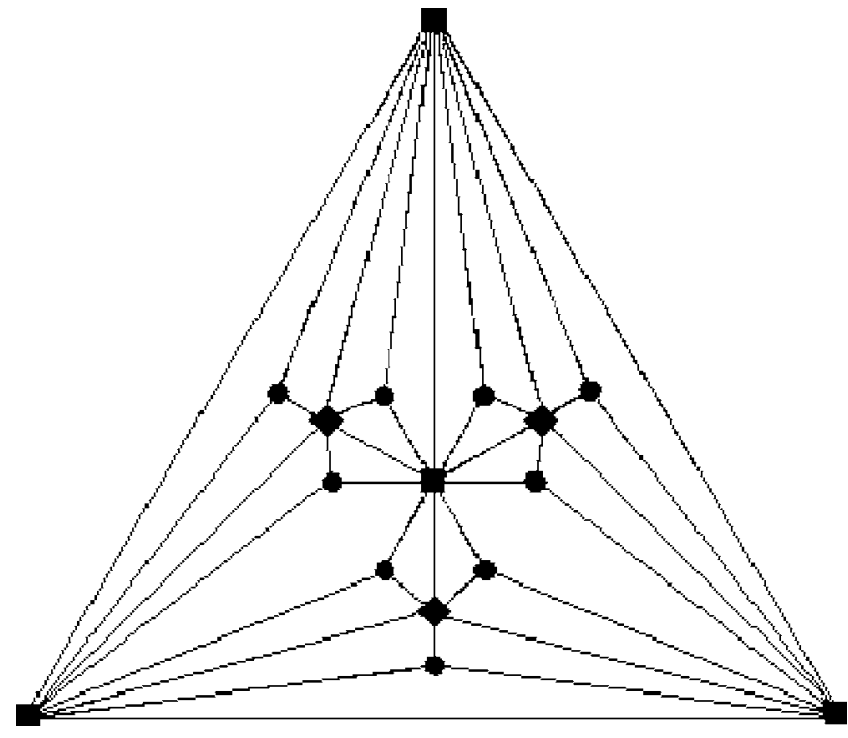

FIG. 1. First three generations of the Apollonian network. Sites represented by squares, diamonds, and circles are introduced in the first, second, and third generations.

in an orbital localized at site $i$; the $\langle i j\rangle$ sum runs over nearestneighbor sites on the AN. ${ }^{19}$

The question of magnetic order in the one-band Hubbard model has been investigated by several authors and much controversy has arisen. In the strong-coupling limit a major part of the ferromagnetic phase is predicted. In this case, for a half-filled band, $U$ is much larger than $t$, and the Hubbard model, using a suitable expansion in perturbation theory, is formally equivalent to the antiferromagnetic Heisenberg model. The Heisenberg exchange parameter $J$ is written in terms of the Hubbard model parameters as $J=-4 t^{2} / U$, where the Hamiltonian of the Heisenberg model is defined by

$$
\mathcal{H}_{e}=-J \sum_{\langle i j\rangle} \vec{S}_{i} \vec{S}_{j},
$$

where $\vec{S}_{i}$ is the total spin-1/2 operator for the $i$ th site. Here we study the anisotropic Heisenberg model to compare the behavior for different values of the anisotropy parameter. ${ }^{18}$

\section{GROUND-STATE PROPERTIES}

We have obtained exact numerical results of the Hubbard model defined on the second generation of the AN, corresponding to seven sites. We computed all the eigenvalues and eigenvectors of the Hamiltonian of Eq. (1) on a basis of states for which the occupation number is diagonal. We consider subspaces of fixed total azimuthal spin operator $S_{Z}$. Thus, the maximum possible dimension of the matrix to diagonalize is 1225 .

Table I shows the results for the spin of the ground state. Until the half-filled band, the occurrence of a ferromagnetic state, where the total spin $S$ is not minimum, can be found only for four electrons. In this case the ground state is threefold degenerate, corresponding to a triplet state of $S=1$. This behavior can be explained considering the spectrum of the
TABLE I. Spin of the ground state as a function of occupation number and $U / t$ on the AN of seven sites.

\begin{tabular}{ll}
\hline \hline$n=2$ & $S=0$, all $U / t$ \\
$n=3$ & $S=1 / 2$, all $U / t$ \\
$n=4$ & $S=1$, all $U / t$ \\
$n=5$ & $S=1 / 2$, all $U / t$ \\
$n=6$ & $S=0$, all $U / t$ \\
$n=7$ & $S=1 / 2$, all $U / t$ \\
$n=8$ & $S=0, U / t<12.5$ \\
& $S=1,12.5<U / t<15.4$ \\
& $S=2,15.4<U / t<20.1$ \\
$n=9$ & $S=3, U / t>y$ \\
$n=10$ & $S=1 / 2$, all $U / t$ \\
& $S=1, U / t<8.44$ \\
$n=11$ & $S=2, U / t>8.44$ \\
$n=12$ & $S=1 / 2, U / t<0.44$ \\
& $S=3 / 2, U / t>0.44$ \\
\hline \hline
\end{tabular}

free electron system. Table II lists the single-electron energies and degeneracies. The double degeneracy in the second level forms a triplet of lowest energy for the four-electron case. For two, three, five, six, and seven electrons the ground state always has minimum spin. We observe that the Coulombian interaction does not favor the occurrence of a highspin ground state. If $U / t$ is strong, the jumps of electrons decrease and a frustrated ordered antiferromagnet is favored. We easily see that the network is not bipartite, so its structure is antiferromagnetically frustrated. We find a similar competition, between interaction and frustration, in the lowtemperature antiferromagnetic state on a triangular lattice. ${ }^{20}$

Above the half-filled band we obtain a ferromagnetic ground state. Nagaoka demonstrated that ferromagnetism is expected for the antiferromagnetically frustrated structures in the half-filled band case with one excess electron and $U$ $\rightarrow \infty .{ }^{21}$ Here, this limit represents the eight-electron case for $U / t \gg 1$. We can see that if $U / t$ increases the alignment of spins also increases, in such a manner that, for $U / t>20.1$, the ferromagnetic state with the maximum total spin is the ground state. For more than eight electrons ferromagnetism is possible, but not stronger than in the case of completely saturated ferromagnetism.

In general, the behavior of short- (or long-) range ordering can be better observed by studying the spin-spin correlation function. Considering the azimuthal spin operator on site $i$ as $S_{i}^{z}=n_{i \uparrow}-n_{i \downarrow}$, the spin-spin correlation function is defined, for $N$ sites, as

TABLE II. Energy levels $E_{i}(i=1,2, \ldots, 7)$ of free electron system $(U / t=0)$ on the AN of seven sites.

\begin{tabular}{lcccc}
\hline \hline$E_{1}$ & $E_{2}=E_{3}$ & $E_{4}$ & $E_{5}=E_{6}$ & $E_{7}$ \\
\hline-4.5114 & -0.6180 & 0.7589 & 1.6180 & 1.7525 \\
\hline \hline
\end{tabular}




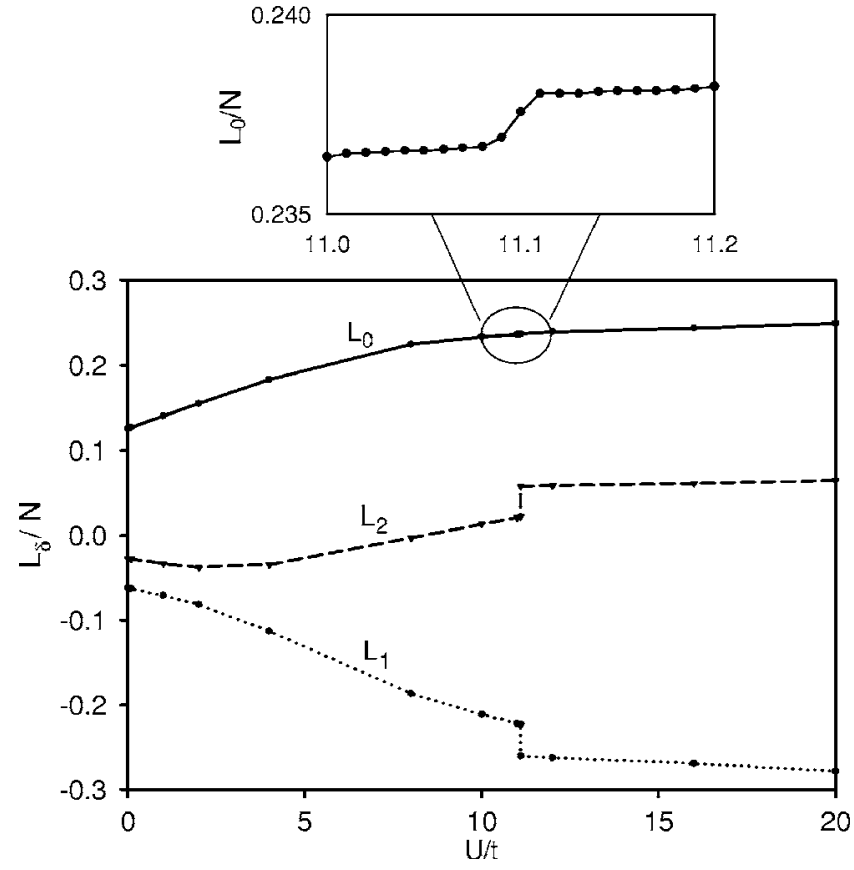

FIG. 2. Ground-state spin-spin correlation function versus $U / t$ for the half-filled band on the AN of seven sites. On top we see a detailed view of the curve for $L_{0}$.

$$
L_{\delta}=\frac{1}{4 N} \sum_{i} S_{i}^{z} S_{i+\delta}^{z} .
$$

The quantity $L_{0}$, called the local moment, depends on the magnitude of the difference between up and down electron spin at each site. It shows the degree of localization of electrons. For a completely localized system in which each site is occupied by a single electron (up or down), $L_{0}=1 / 4$, while for a noninteracting system $L_{0}=1 / 8 . L_{\delta}(\delta \neq 0)$ is the correlation between the electron spins at different sites. They are related to the magnetic ordering. The results for the groundstate spin-spin correlation function versus $U / t$ in the halffilled band case are presented in Fig. 2. $L_{0}$ gradually increases if $U / t>0$ increases, i.e., electrons gradually localize. $L_{1}$ has a negative sign and increases if $U / t>0$ increases and $L_{2}$ has a critical value for $U / t=8$ where the behavior changes from a negative to a positive sign. For $U / t>8, L_{2}$ is positive, $L_{1}$ is negative, and an antiferromagnetic order appears.

For $U / t=11.1$ there is a change in the spatial symmetry of the ground state. It is important to observe the discontinuous behavior of $L_{\delta} \cdot{ }^{12}$ Recent studies of the metal-insulator transition on the triangular lattice for the half-filled Hubbard model revealed a critical interaction $U / t=12$ for a Mott metal-insulator transition. ${ }^{10,11}$ Reference 10 uses dynamical mean-field theory and quantum Monte Carlo simulations to investigate the triangular lattice at finite temperature. Their results are consistent with the ones of Ref. 11 from the Kotliar-Ruckenstein slave-boson technique and an exact numerical diagonalization on a 12-site lattice at zero temperature. The AN has a structure comparable to the frustation effect of the tringular lattice. The change in the spatial symmetry of the ground state for $U / t=11.1$ on the AN has a similar feature with the exact-diagonalization results of Ref. 11 on a triangular lattice. This suggests a first-order transition between the metal and insulator on the $\mathrm{AN}$ at $U / t$ $=11.1$ at zero temperature. For $U / t \gg 1, L_{0}$ is approximately equal to $1 / 4$ and we can conclude that each site is occupied by just one electron. The ground state has minimum spin $S$ $=1 / 2$. We cannot analyze if larger ANs present a ferrimagnetic order at temperature $T=0$. For $U / t<8, L_{2}$ is negative and the magnetic behavior of the ground state is more complex.

We analyze the wave function of the ground state, defining $P_{k}=\left|\Psi_{k 0}\right|^{2}$, where $\left|\Psi_{k 0}\right|$ is the $n$th component of the ground eigenstate. We define the total antiferromagnetic (AF) configuration $(k=\mathrm{AF})$ such that sites introduced in the first generation on the AN (represented by squares in Fig. 1) are occupied by electrons of one type of spin and sites introduced in the second generation (represented by diamonds in Fig. 1) are occupied by electrons of the opposite spin. We observe that $P_{\mathrm{AF}} / P_{k}>2$ for $U / t=0$ and increases to $P_{\mathrm{AF}} / P_{k}>15$ if $U / t=8$, for all $k \neq \mathrm{AF}$, i.e., the total antiferromagnetic configuration $P_{\mathrm{AF}}$ is approximately two times for $U / t=0$ and 15 times for $U / t=8$ more probable than any another configuration.

We can construct a physical picture where the ground state has an antiferromagnetic order (frustrated) at any $U / t$. In contrast, we shall see in the present work, using the realspace renormalization group approach for the antiferromagnetic Heisenberg model on the AN, that the strong-coupling Hubbard model, at finite temperature, does not have an ordered phase. The above observations suggest that, in the thermodynamic limit, the Hubbard model on the AN, at any $U / t>0$, has an antiferromagnetic order with a metalinsulator transition for $U / t=11.1$ at temperature $T=0$ and a disordered phase for any finite temperature, where we were not able to observe the Mott transtion.

\section{THERMODYNAMIC PROPERTIES}

On one hand, we study the thermodynamic properties of the half-filled band Hubbard model using small-cluster exact-diagonalization calculations in the grand canonical ensemble. ${ }^{13}$ We have calculated all eigenvalues and eigenfunctions for an AN of seven sites. On the other hand, we study lattices of 63 and 124 sites using the grand canonical quantum Monte Carlo method. ${ }^{14,15}$ We used a discrete Hubbard-Stratonovich transformation to convert the problem into one of free particles interacting with a time-dependent Ising field, together with an exact updating algorithm for the fermion Green's function to compute the relative weights of the Ising configurations.

We consider several values of $U / t$ for all the studied thermodynamic properties. We determine with considerable accuracy the temperature dependence of the spin-spin correlation functions, spin susceptibility, specific heat, double occupancy, and one-electron transfer.

The temperature dependence of $L_{0}, L_{1}$, and $L_{2}$ for some typical values of $U / t$ is shown in Figs. 3(a)-3(c). If $U / t$ increases, $L_{0}$ gradually increases for $U / t>0$, indicating that electrons are gradually localizing. At high temperature $L_{0}$ 

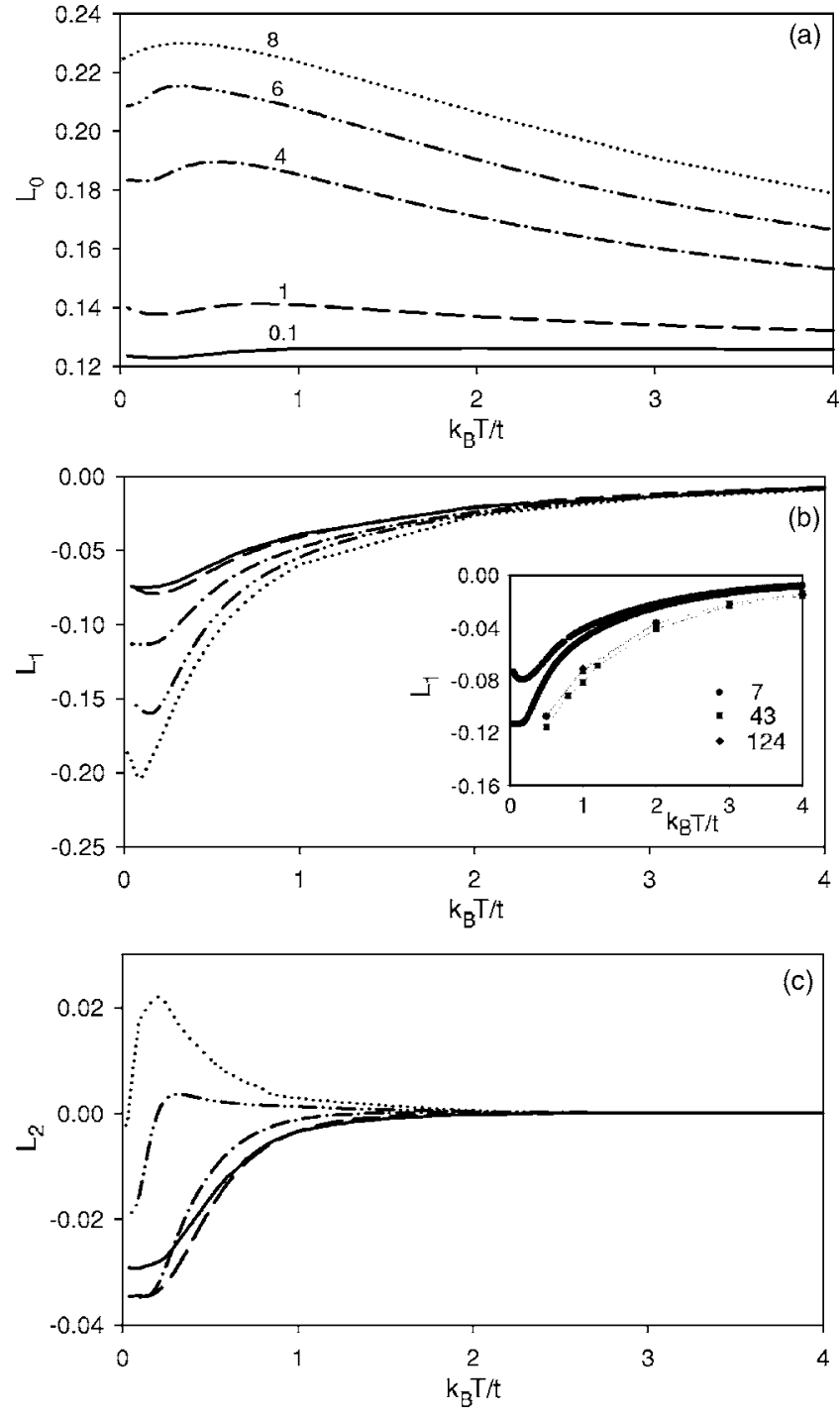

FIG. 3. Correlation functions $L_{\delta}$ vs temperature with $U / t=0.1$, 1, 6, and 8 for the AN of seven sites. (a) $L_{0}$; (b) $L_{1}$; (c) $L_{2}$. In the inset of (b), $L_{1}$ with $U / t=1$ (black) and $U / t=4$ (gray) for 7, 43, and 124 sites on the AN using the Monte Carlo method.

gradually decreases and electrons gradually delocalize. At very low temperatures, the temperature dependence with negative signs of $L_{1}$ induces an antiferromagetic ordering. The inset in Fig. 3(b) shows Monte Carlo results for the AN with 7, 43, and 124 sites and $U / t=1$ and 4. Results for 43 and 124 sites do not reveal any new behavior; however, the antiferromagetic ordering increases. We observe that the properties obtained for seven sites must be qualitatively equivalent to those for larger ANs. We observe a competition between interaction and frustration in the ordered antiferromagnetic state by the analysis of $L_{2}$. For small or intermediate $U / t$, the negative sign of $L_{2}$ is a result of the frustrated structure of the AN, disfavoring the antiferromagnetic order. For $U / t \gg 1, L_{2}$ has a positive sign, showing that the strong electron interaction favors the antiferromagnetic order.

Figure 4 shows the temperature dependence of the magnetic susceptibility $\chi$. The Curie-Weiss behavior, $\chi \propto 1 /(T$ $-\theta)$, is observed for high temperatures. For small and inter-

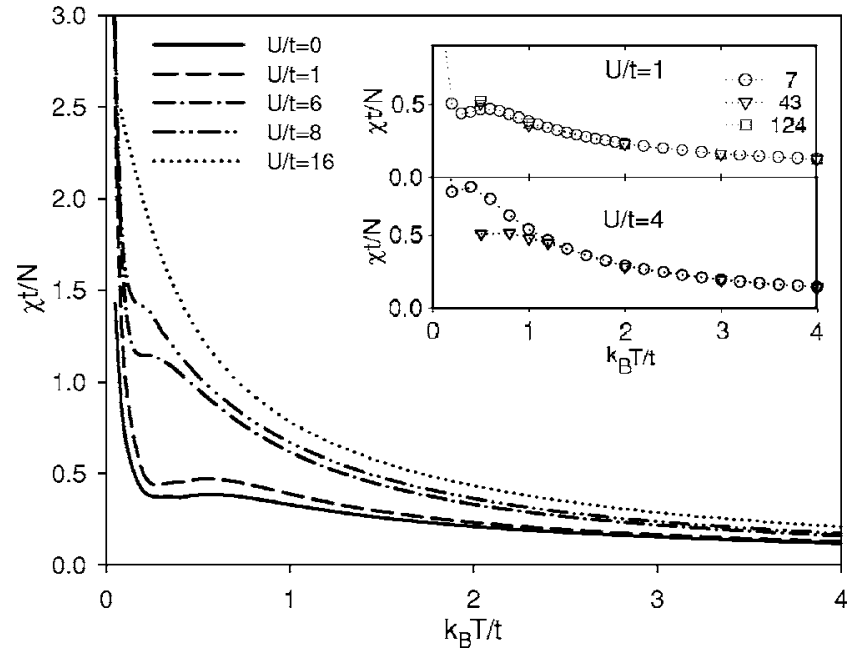

FIG. 4. Magnetic susceptibility as a function of temperature with $U / t=0,0.5,4$, and 8 for the $\mathrm{AN}$ of seven sites. Inset: Magnetic susceptibility with $U / t=1$ and 4 for 7,43 , and 124 sites on the AN using the Monte Carlo method.

mediate $U / t$ the curves present a peak. The temperature associated with the peak of $\chi$ corresponds approximately to the rapid decay of $L_{1}$ and $L_{2}$, and is a consequence of the collective excitations that lead to the destruction of the antiferromagetic order. ${ }^{12}$ Here the ground state has spin $S=1 / 2$ and $\chi$ must go to infinity at temperature $T=0$. The susceptibility $\chi$ with $U / t=1$ and 4 for 7,43 , and 124 sites on the AN using the Monte Carlo method is shown in the inset of Fig. 4.

A comparison between results for different clusters shows a good agreement at large temperatures, but not at low temperatures. This result may be explained considering that we are studying finite clusters of odd number of sites and electrons. In these cases, $\chi$ must go to infinity at $T=0$. As a consequence of the finite-size effect, if we increase the size of a system with an odd number of sites and minimum spin $S=1 / 2$, the relevance of the spin $S=1 / 2$ for the magnetic susceptibility decreases and the values of $\chi$ at low temperature must also decrease. The finite-size effect for $\chi$ indicates that the $\mathrm{AN}$ does not present a ferrimagnetic order in the thermodynamic limit.

The standard sign problem of the quantum Monte Carlo method $^{14}$ is relevant. The absence of the particle-hole symmetry from frustrated lattices, like the AN and the triangular lattice, implies a sign problem even at half filling. Due to this problem, we cannot go to low $T$ and large $U / t$. However, the finite-size effect becomes in general less important as $U$ increases. ${ }^{10}$

The temperature dependence of the specific heat is shown in Fig. 5 for typical values of $U / t$. For small values of $U / t$ there is a peak in the specific heat. On increasing $U / t$, the peak splits into two, which reflects a rearrangement of the fermionic structure in the system. The low-temperature peak arises due to low-lying collective excitations, while the hightemperature broad peak comes from single-particle excitations. This behavior is quite general and has been noticed for different structures. ${ }^{12,13}$

Next, we study the thermal average of the double occupancy $d=(1 / N) \sum_{i} n_{i \uparrow} n_{i \downarrow}$ and of the one-electron transfer defined by 


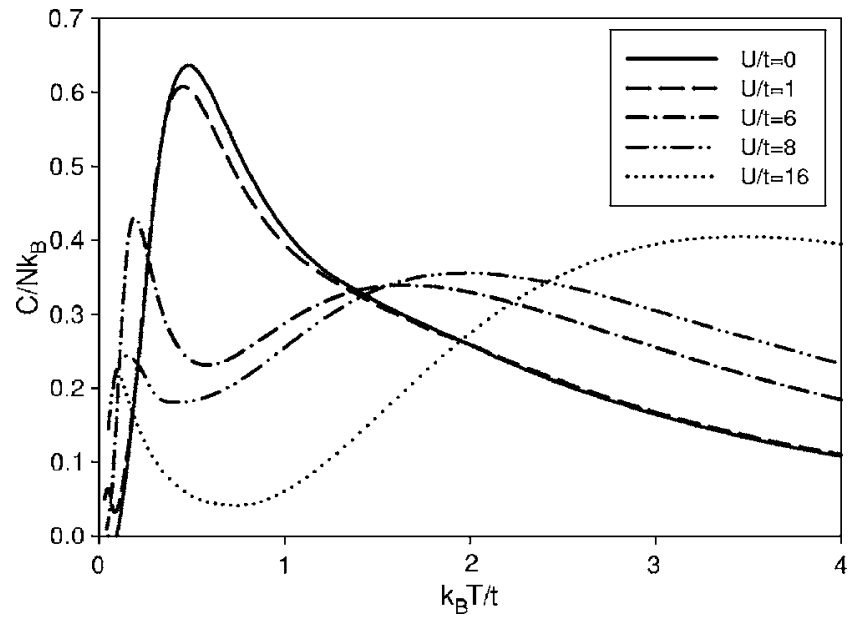

FIG. 5. Specific heat $C / N k_{B}$ as a function of temperature with $U / t=0,0.5,4$, and 8 for the AN of seven sites.

$$
j=\sum_{\langle i j\rangle \alpha} c_{i \alpha}^{\dagger} c_{j \alpha}=\frac{U d-\mathcal{H}}{t} .
$$

Figure 6 shows the temperature dependence of $j$ and $d$ (inset) with $U / t=0.1,1$ and 4 for the AN with 124 sites. Clearly these functions are related to the local moment. It is easy to show that for the half-filled band $d=\left(1-4 L_{0}\right) / 2$. If $U / t$ increases, the local moment increases [see Fig. 3(a)] and the double occupation and one-electron transfer decrease. In the strong-coupling limit $(U / t \gg 1)$ each site is occupied by just one electron and the electronic itinerancy vanishes. This region is characterized by localized magnetism and we can apply the Heisenberg model.

\section{LOCALIZED MAGNETISM: RENORMALIZATION GROUP APPROACH}

The fractal and self-similar nature of scale-invariant networks, generated through a recursive procedure, offers an appropriate way to get rigorous results at the phase transi-

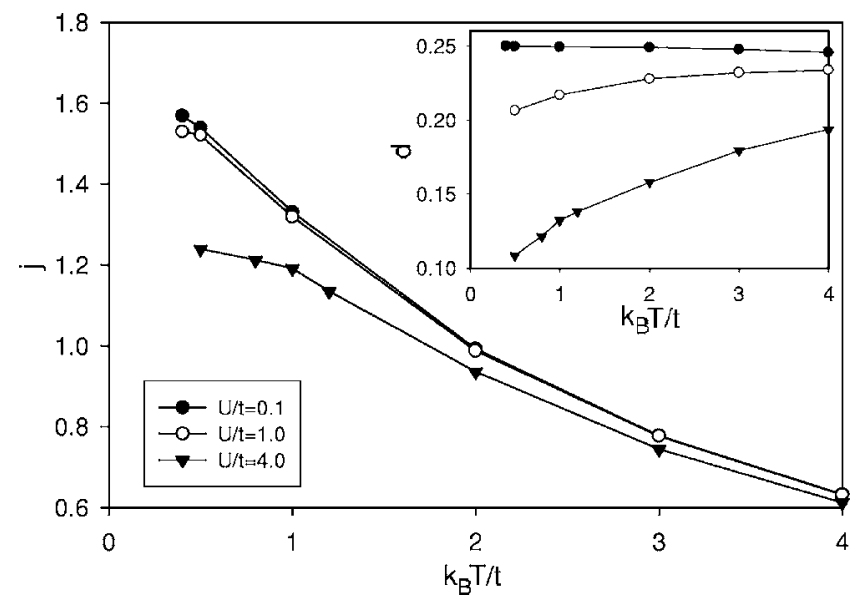

FIG. 6. One-electron transfer $j$ as a function of temperature with $U / t=0.1,1$, and 4 for the AN of 124 sites. Inset: Double occupancy $d$ as a function of temperature.

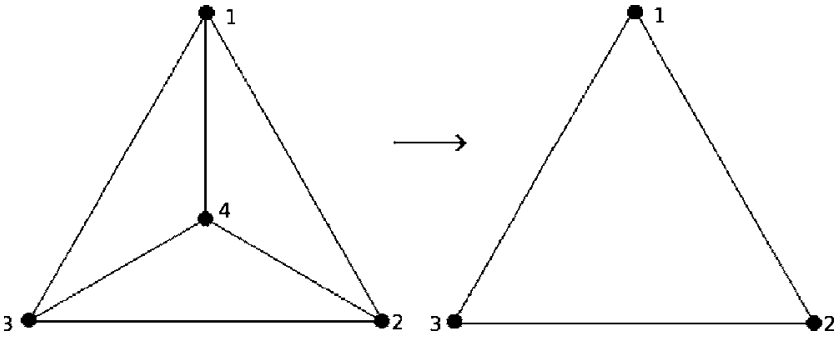

FIG. 7. Renormalization group transformation associated with the AN.

tion. The difficulty with the spin-decimation process, which leads to the renormalization group equation as found on Bravais lattices due to parameter proliferation, disappears on scale-invariant networks. ${ }^{23,24}$ Because fractal lattices are characterized by dilation invariance but do not have translational invariance, the study on these networks can with restriction be used to model Bravais lattices. Particularly selfsimilar networks can mimic Bravais lattices for some magnetic models, providing equal critical temperatures and exponents. ${ }^{24,25}$

The real-space renormalization group ( $R G)$ approach has been applied with success to the study of the anisotropic spin-1/2 Heisenberg model on self-dual hierarchical lattices. ${ }^{16-18}$ Here, we apply this analysis on an AN. It is defined by the cluster transformation process, preserving the Hamiltonian form, as illustrated in Fig. 7.

The dimensionless Hamiltonian is defined by

$$
h=-\beta \mathcal{H}=\frac{4 J}{k_{B} T} \sum_{\langle i j\rangle}\left[(1-\Delta)\left(S_{i}^{x} S_{j}^{x}+S_{i}^{y} S_{j}^{y}\right)+S_{i}^{z} S_{j}^{z}\right],
$$

where $\beta \equiv 1 / k_{B} T,\langle i j\rangle$ denotes first-neighboring lattice sites, $\Delta$ is the anisotropy parameter, and $S_{i}^{\alpha}\{\alpha=x, y, z\}$ is the $\alpha$ spin-1/2 operator on site $i$. The RG recurrence equation is obtained by imposing

$$
\exp \left(h_{123}^{\prime}+C\right)=\underset{\text { site } 4}{\operatorname{Tr}} \exp \left(h_{1234}\right)
$$

where $h_{1234}$ and $h_{123}^{\prime}$ are, respectively, the Hamiltonians of the four-site cluster and of the renormalized three-site cluster, shown in Fig. 7. To make the RG equation possible, an additive constant $C$ has been included. The RG Eq. (6) establishes the relation between the set of parameters $(J, \Delta)$ and the set of renormalized parameters $\left(J^{\prime}, \Delta^{\prime}\right)$. The noncommutativity between the Hamiltonians associated with neighboring clusters is neglected, and therefore our results are approximations for all temperatures, being asymptotically exact only at high temperatures. ${ }^{17}$

Defining $K \equiv J / k_{B} T$ and expanding $\exp \left(h_{123}^{\prime}\right)$ as

$$
\begin{aligned}
\exp \left(h_{123}^{\prime}+C\right)= & a^{\prime}+4 b^{\prime}\left(S_{1}^{x} S_{2}^{x}+S_{1}^{y} S_{2}^{y}+S_{1}^{x} S_{3}^{x}+S_{1}^{y} S_{3}^{y}+S_{2}^{x} S_{3}^{x}\right. \\
& \left.+S_{2}^{y} S_{3}^{y}\right)+4 c^{\prime}\left(S_{1}^{z} S_{2}^{z}+S_{1}^{z} S_{3}^{z}+S_{2}^{z} S_{3}^{z}\right),
\end{aligned}
$$

we obtain that

$$
4 K^{\prime}=\ln \left(\frac{a^{\prime}+3 c^{\prime}}{a^{\prime}-c^{\prime}-2 b^{\prime}}\right)+\frac{1}{3} \ln \left(\frac{c^{\prime}-a^{\prime}+2 b^{\prime}}{c^{\prime}-a^{\prime}-4 b^{\prime}}\right),
$$




$$
\begin{gathered}
6 K^{\prime} \Delta^{\prime}=\ln \left(\frac{c^{\prime}-a^{\prime}-4 b^{\prime}}{c^{\prime}-a^{\prime}+2 b^{\prime}}\right), \\
C=-3 k^{\prime}+\ln \left(a^{\prime}+3 c^{\prime}\right) .
\end{gathered}
$$

Analogously,

$$
\begin{aligned}
\exp \left(h_{1234}\right)= & a+4 \sum_{(i<j)}\left[b\left(S_{i}^{x} S_{j}^{x}+S_{i}^{y} S_{j}^{y}\right)+c S_{i}^{z} S_{j}^{z}\right] \\
& +16 \sum_{(i<j) \neq(k<l)}\left[d\left(S_{i}^{x} S_{j}^{x}+S_{i}^{y} S_{j}^{y}\right) S_{k}^{z} S_{l}^{z}+g\left(S_{i}^{x} S_{j}^{x}\right.\right. \\
& \left.\left.+S_{i}^{y} S_{j}^{y}\right)\left(S_{k}^{x} S_{l}^{x}+S_{k}^{y} S_{l}^{y}\right)\right]+16 f S_{1}^{z} S_{2}^{z} S_{3}^{z} S_{4}^{z}
\end{aligned}
$$

where $a, b, c, d, g$, and $f$ are functions of $K$ and $\Delta$. It is easy to see from Eq. (6) that $a^{\prime}=2 a, b^{\prime}=2 b$, and $c^{\prime}=2 c$. The set of parameters $\left(K^{\prime}, \Delta^{\prime}\right)$ as functions of $(K, \Delta)$ can be determined by diagonalizing $h_{123}$ and $h_{1234}$ and using Eqs. (6)-(9). After some calculus we obtain the analytical RG equation as

$$
\begin{gathered}
a^{\prime}=\frac{1}{4}\left(e^{6 K}+e^{6 K \Delta}+3 e^{-2 K \Delta}+\frac{e^{-2 K}}{2}\left(3+e^{8 K \Delta}+2 e^{-4 K \Delta}\right)\right) \\
b^{\prime}=\frac{1}{4}\left(\frac{e^{6 K \Delta}-e^{-2 K \Delta}}{2}+\frac{e^{-2 K}}{3}\left(e^{8 K \Delta}-e^{-4 K \Delta}\right)\right) \\
c^{\prime}=\frac{1}{4}\left(e^{6 K}-\frac{e^{-2 K}}{6}\left(3+e^{8 K \Delta}+2 e^{-4 K \Delta}\right)\right),
\end{gathered}
$$

where $K^{\prime}$ and $\Delta^{\prime}$ are functions of $c^{\prime}, a^{\prime}, b^{\prime}$ given by Eq. (8). We observe that the Ising and isotropic Heisenberg models are mapped into themselves. The RG recurrence is simplified as

$$
k^{\prime}=\frac{1}{4} \ln \left(\frac{1+e^{6 K}}{1+e^{-2 K}}\right)
$$

and

$$
k^{\prime}=\frac{1}{6} \ln \left(\frac{3+5 e^{8 K}}{6+2 e^{-4 K}}\right)
$$

corresponding to the Ising $(\Delta=1)$ and the isotropic Heisenberg $(\Delta=0)$ limits, respectively.

Figure 8 shows the phase diagram in the $\left(k_{B} T / J, \Delta\right)$ space. The results for the ferromagnetic case $\left(k_{B} T / J>0\right)$ do not have a relation with the Hubbard model. However, considering that they represent a situation that has not yet been explored, we think these results are important to shed light on the understanding of the magnetism on the AN. In the ferromagnetic case, we always observe the existence of ferromagnetism $(F)$ independent of $\Delta$. This result has been found on the AN for the Ising model using the transfer matrix method ${ }^{8}$ and on other scale-free lattices. ${ }^{22}$ Those results agree very well with ours. Here, we observe that the quantum fluctuations of the $X Y$ part of our Hamiltonian do not destroy this ordering generated by the topology of the AN.

In the antiferromagnetic case $\left(k_{B} T / J<0\right)$, we verify the absence of the ordered phase for all $\Delta$ at finite $T$. Again, the results obtained are similar to and consistent with the Ising limit. ${ }^{8}$ The antiferromagnetic case represents the strong-
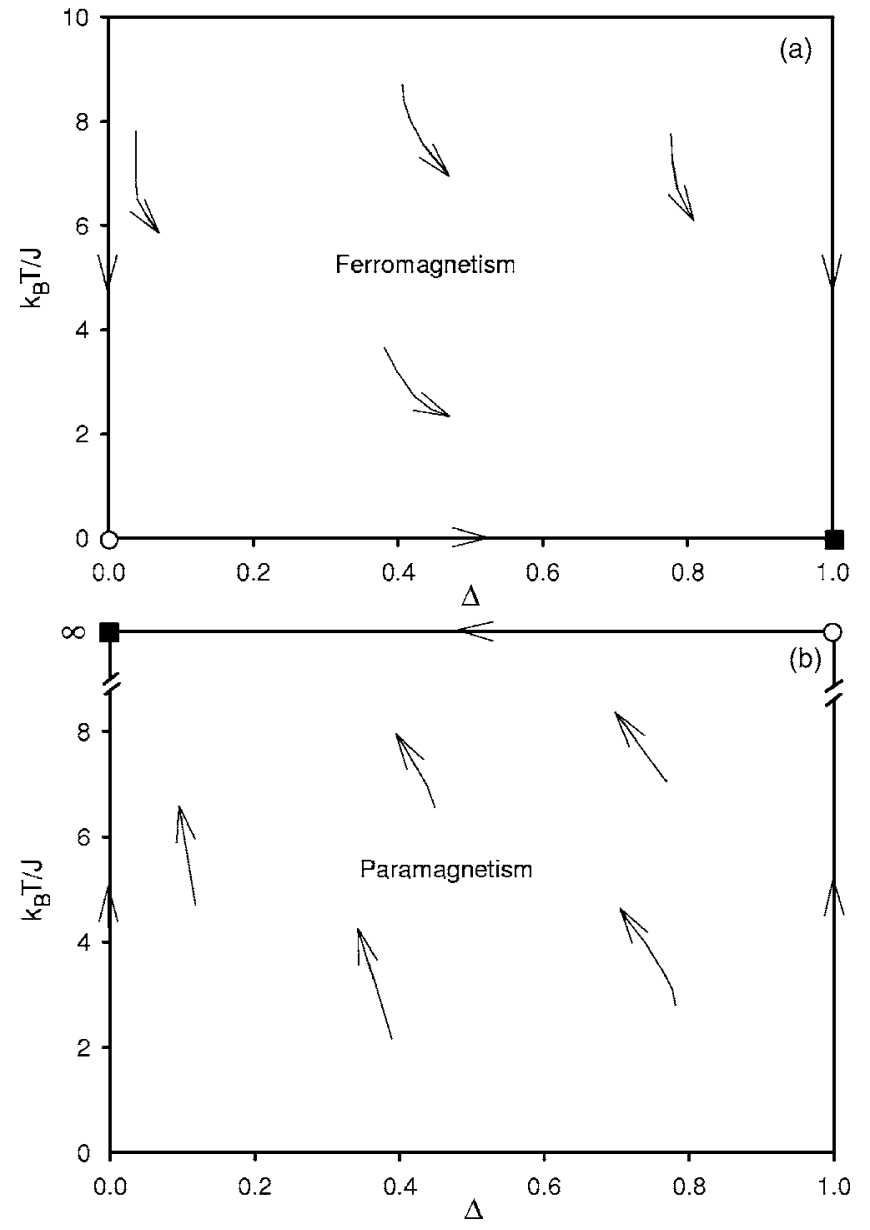

FIG. 8. Flow diagram for the Apollonian cell of Fig. 7. Open circles and full squares respectively denote the semistable and fully stable fixed points. (a) Ferromagnetic case $(J>0)$; (b) antiferromagnetic case $(J<0)$.

coupling limit of the Hubbard model. The strong-coupling limit is the region where a major part of the ordered phase has been found. ${ }^{21}$ This suggests a tendency toward the nonordered phase in the Hubbard model for any $U / t$ at finite temperature on the AN.

\section{CONCLUSIONS}

In conclusion, we have analyzed strongly correlated electron systems on the AN. The ground state and thermodynamic properties of the Hubbard model have been studied using exact diagonalization calculations and quantum Monte Carlo simulations. The dependence on the ratio $U / t$ of the specific heat, the magnetic susceptibility, the spin-spin correlation function, the double occupancy, and the one-electron transfer support several types of magnetic behavior for finite AN clusters. We have also studied the magnetic properties of the anisotropic spin-1/2 Heisenberg model on the AN using the real-space RG approach. For ferromagnetic coupling, we always observe the existence of ferromagnetism independent of temperature and the anisotropy parameter $\Delta$. As opposed to other structures, ${ }^{17,18}$ the topology of the AN favors ferromagnetic order, and the quantum fluctuations do not destroy 
this ordering. For antiferromagnetic coupling, we find a paramagnetic phase for all $\Delta$ at finite $T$. A similar result has been found on the AN for the Ising model using the transfer matrix method. ${ }^{8}$ Consistent with a paramagnetic metalparamagnetic insulator transition at $U / t=12$ obtained recently on the triangular lattice, ${ }^{10}$ our results suggest an antiferromagnetic order with a metal-insulator transition for $U / t=11.1$ at temperature $T=0$ and a tendency toward a nonordered phase in the half-filled Hubbard model for any $U / t$.
This indicates that the spectral dimension ${ }^{5}$ controls the magnetic behavior on the AN.

\section{ACKNOWLEDGMENTS}

The authors thank P. G. Lind and E. Parteli for their generous help. This work was supported by CNPq (Brazil), DAAD (Germany), and the Max Planck Society Research Award.
${ }^{1}$ R. Albert and A. L. Barabási, Rev. Mod. Phys. 74, 47 (2002).

${ }^{2}$ S. N. Dorogovtsev and J. F. F. Mendes, Evolution of Network From Biological Nets to the Internet and WWW (Oxford University Press, Oxford, 2003); S. N. Dorogovtsev and J. F. F. Mendes, Adv. Phys. 51, 1079 (2002).

${ }^{3}$ A. L. Barabási and R. Albert, Science 286, 509 (1999).

${ }^{4}$ G. R. Newkome, P. Wang, C. N. Moorefield, T. J. Cho, P. Mohapatra, S. Li, S. H. Hwang, O. Lukoyanova, L. Echegoyen, J. A. Palagallo, V. Iancu, and S. W. Hla, Science 312, 1782 (2006).

${ }^{5}$ M. Schreiber and H. Grussbach, Phys. Rev. Lett. 76, 1687 (1996)

${ }^{6}$ M. Sade, T. Kalisky, S. Havlin, and R. Berkovits, Phys. Rev. E 72, 066123 (2005).

${ }^{7}$ J. S. Andrade, Jr., H. J. Herrmann, R. F. S. Andrade, and L. R. da Silva, Phys. Rev. Lett. 94, 018702 (2005).

${ }^{8}$ R. F. S. Andrade and H. J. Herrmann, Phys. Rev. E 71, 056131 (2005)

${ }^{9}$ J. P. K. Doye and C. P. Massen, Phys. Rev. E 71, 016128 (2005).

${ }^{10}$ K. Aryanpour, W. E. Pickett, and R. T. Scalettar, Phys. Rev. B 74, 085117 (2006).

${ }^{11}$ M. Capone, L. Capriotti, F. Becca, and S. Caprara, Phys. Rev. B 63, 085104 (2001).

${ }^{12}$ J. Callaway, D. P. Chen, and R. Tang, Phys. Rev. B 35, 3705
(1987); J. Callaway, D. P. Chen, and Y. Zhang, ibid. 36, 2084 (1987).

${ }^{13}$ C. A. Macedo and A. M. C. de Souza, Phys. Rev. B 65, 153109 (2002).

${ }^{14}$ J. E. Hirsch, Phys. Rev. B 31, 4403 (1985).

${ }^{15}$ C. A. Macedo and A. M. C. Souza, Physica B 354, 290 (2004); A. M. C. Souza and C. A. Macedo, J. Magn. Magn. Mater. 226, 2026 (2001).

${ }^{16}$ A. O. Caride, C. Tsallis, and S. I. Zanette, Phys. Rev. Lett. 51, 145 (1983).

${ }^{17}$ A. M. Mariz, C. Tsallis, and A. O. Caride, J. Phys. C 18, 4189 (1985).

${ }^{18}$ A. M. C. de Souza, Phys. Rev. B 48, 3744 (1993).

${ }^{19}$ R. F. S. Andrade and J. G. V. Miranda, Physica A 356, 1 (2005).

${ }^{20}$ A. Singh, Phys. Rev. B 71, 214406 (2005).

${ }^{21}$ Y. Nagaoka, Phys. Rev. 147, 392 (1966).

${ }^{22}$ A. Aleksiejuka, J. A. Holyst, and D. Stauffer, Physica A 310, 260 (2002).

${ }^{23}$ Z. R. Yang, Phys. Rev. B 38, 728 (1988).

${ }^{24}$ M. Kaufman and R. B. Griffiths, Phys. Rev. B 24, 496 (1981).

${ }^{25}$ A. M. C. de Souza, C. Tsallis, and A. M. Mariz, Phys. Rev. B 47, 11940 (1993). 